\title{
Análise da correlação entre resistência à compressão axial e à tração na flexão em pavimentos rígidos
}

Luciana Rabelo de Brito (iD lucianaa_rabelo@hotmail.com Engenheira Civil. Governo do Estado do Ceará

Antônia Fabiana Marques Almeida iD fabiana@det.ufc.br Universidade Federal do Ceará (UFC)

Francisco Heber Lacerda de Oliveira (iD heber@det.ufc.br Universidade Federal do Ceará (UFC)

\section{Analysis of correlation between axial compression resistance and three-point flexural resistance in rigid pavements}

\author{
Análisis de la correlación entre resistencia a la compresión \\ axial y a la tracción en la flexión en suelos rígidos
}

\section{Analyse de corrélation entre la résistance à la compression axiale et la résistance à la traction en flexion dans les chaussées rigides}

\section{Resumo}

O concreto de cimento Portland é um material compósito que tem como principal característica a resistência mecânica à compressão. Porém, quando se trata de pavimento rígido de concreto simples, outra propriedade mecânica que assume destaque é a resistência à tração na flexão. Essa propriedade é uma forma indireta de obter a tração direta, pois o seu ensaio não é muito empregado devido ao dispositivo que prende o corpo de prova ao equipamento inserir cargas que afetam no resultado obtido. Essa obtenção indireta é normalizada e considera que a tração direta equivale a $70 \%$ do valor obtido da tração na flexão. Como ensaios de resistência à compressão são mais realizados, a mesma norma prevê a possibilidade de obter o valor da tração direta através do $f_{c k}$. Como o concreto é influenciado por vários fatores externos e internos, que muitas vezes não há como mensurar, tem-se a premissa que essa equação não é válida para todos os conjuntos de dados. Nesse sentido, este trabalho buscou, com um conjunto de dados obtidos do controle tecnológico da execução de placas de concreto simples do Quarto Anel Rodoviário, em Fortaleza, avaliar a correlação existente entre esses parâmetros. Os resultados analisados encontraram uma equação que difere da prevista em norma. Contudo os erros calculados com a aplicação das duas equações são próximos, fazendo com que a equação ora existente possa ser aplicada.

Palavras-chave: Pavimento. Concreto. Compressão. Tração. FlexãoCorrelação.

\begin{abstract}
Portland cement concrete is a composite material whose main characteristic for structures is mechanical resistance to compression. However, when it comes to concrete pavement, another mechanical property that stands out is the flexural resistance. This property is an indirect way of obtaining direct tensile the strength, because its test is not much used due to the device that holds the test body to the equipment to insert loads that affect the result obtained. This indirect gain is normalized, and it considers the pure tensile equals to $70 \%$ of the obtained value of the flexural test. As tests of compressive strength are more accomplished, the same norm predicts the possibility of obtaining the value of the pure tensile through $f_{c k}$. As concrete is influenced by several external and internal factors, which one often does not have to measure, one assumes that this equation is not valid for all datasets. In this sense, this paper sought, with a set of data obtained from the technological control of the execution of simple concrete slabs of the Fourth Ring Road in Fortaleza, to evaluate the correlation between these parameters. The analyzed results found an equation that differs
\end{abstract}


from that predicted in standard. However, the errors calculated with the application of the two equations are close, so that the existing equation can be applied.

Keywords: Pavement. Concrete. Compression. Tensile. Flexura. Correlation.

\begin{abstract}
Resumen
El hormigón de cemento Portland es un material compuesto que tiene como principal característica la resistencia mecánica a la compresión. Sin embargo, cuando se trata de suelo rígido de hormigón simple, otra propiedad mecánica que adquiere enfoque es la resistencia a la tracción en la flexión. Esta propiedad es una forma indirecta de obtener la tracción directa, porque su ensayo no es muy empleado debido al dispositivo que sujeta el cuerpo de prueba al equipo introducir cargas que afectan en el resultado obtenido. Esta obtención indirecta es normalizada y considerada que la tracción directa equivale a $70 \%$ del valor obtenido de la tracción en la flexión. Como ensayos de resistencia a la compresión son realizados con más frecuencia, la misma norma prevé la posibilidad de obtener el valor de la tracción directa por medio del $f_{c k}$. Como el hormigón sufre distintas influencias de factores internos y externos, que muchas veces no hay como mensurar, se tiene la premisa que esta ecuación no es válida para todo el conjunto de datos. En este sentido, este trabajo busco, con un conjunto de datos obtenidos del control tecnológico de la ejecución de placas de hormigón simple del Cuarto Anel de Carretera, en Fortaleza, evaluar la correlación existente entre estos parámetros. Los resultados analizados encontraron una ecuación que es diferente de la prevista en norma. Pero los errores calculados con la aplicación de las dos ecuaciones son próximos, haciendo con que la ecuación ora existente pueda ser aplicada.
\end{abstract}

Palabras-clave: Suelo. Hormigón. Compresión. Tracción. Flexión. Correlación.

\title{
Résumé
}

Le béton de ciment Portland est un matériau composite dont la principale caractéristique est la résistance mécanique à la compression. Cependant, lorsqu'il s'agit d'un revêtement dur en béton commun, une autre propriété mécanique qui se distingue est la résistance à la traction en flexion. Cette propriété est un moyen indirect d'obtenir une traction directe, lorsque son test n'est pas largement employé en raison du dispositif qui maintient l'échantillon adhéré à l'équipement, puisqu'il insère des charges qui affectent le résultat obtenu. Cette obtention indirecte est normalisée et considère que la traction directe équivaut à $70 \%$ de la valeur obtenue en traction en flexion. Comme les essais de résistance à la compression sont plus fréquemment effectués, la même norme prévoit la possibilité d'obtenir la valeur de traction directe par le Fck. Le béton est influencé par un certain nombre de facteurs externes et internes, qui souvent ne peuvent pas être mesurés. Donc, on suppose que cette équation n'est pas valable pour tous les ensembles de données. En ce sens, ce travail a cherché évaluer la corrélation entre ces paramètres, en utilisant un ensemble de données obtenu à partir du contrôle technologique de l'exécution de simples dalles de béton du quatrième périphérique, à Fortaleza, dans l'État du Ceará, Brésil. Les résultats analysés ont révélé une équation différente de celle prévue comme standard. Cependant, les erreurs calculées en appliquant les deux équations sont proches, de sorte que l'équation existante peut être appliquée.

Mots-clés: Chaussé. Béton. Compression. Traction. Flexion Corrélation.

\section{Introdução}

O pavimento rígido é uma opção para construção de camadas de rolamento de vias de tráfego. É fabricado com concreto de cimento Portland, e pode ser executado em placas simples, armadas e protendidas. No Brasil, de acordo com CNT (2017), somente $1 \%$ das vias pavimentadas utilizam essa solução. 
O pavimento de concreto, devido à sua elevada rigidez, diferente do pavimento flexível, é capaz de absorver quase totalmente as tensões aplicadas pelas cargas impostas pelo tráfego, aumentando a vida útil da estrutura. Além disso, o pavimento rígido promove estruturas mais delgadas, pois funciona com caráter estrutural, apresentando melhor capacidade de carga, e ainda pode eliminar a camada de base, já que pode atuar como base e revestimento simultaneamente.

Apesar de ter um custo de implantação maior que o pavimento flexível, o pavimento rígido tem baixa necessidade de manutenção ao longo de sua vida útil (BIANCHI et al., 2008). Com base nessas considerações, sabe-se que é uma opção viável para vias que apresentam alto volume de tráfego e para locais onde as cargas são elevadas, como em vias utilizadas para transportar cargas.

Uma das premissas determinadas por DNIT (2005) para pavimentos de concreto simples é o atendimento da resistência à tração na flexão mínima de 4,5 MPa. Para conhecer esse parâmetro, podem ser realizados ensaios com o uso de corpos de prova prismáticos moldados em campo. Porém, a ABNT (2014) prevê a possibilidade de obtenção desse parâmetro através de uma correlação com a resistência à compressão axial.

Diante do exposto, este trabalho tem como objetivo verificar, com os dados da obra de requalificação e duplicação da Rodovia Quarto Anel Viário, localizado na Região Metropolitana de Fortaleza, qual a correlação entre os parâmetros descritos anteriormente e se corresponde ao previsto por ABNT (2014).

\section{Referencial Teórico}

O concreto é um material compósito, de estrutura complexa, obtido a partir da mistura de um aglomerante hidráulico (o mais comum é o cimento Portland, que gera o concreto de cimento Portland), agregados graúdo e miúdo, e água, podendo, ou não, conter aditivos e/ou adições. É formado por três fases: agregado, pasta e zona de transição. Tem como principal característica a capacidade de resistência aos esforços de compressão, que é influenciada por inúmeros fatores, tais como o fator água/cimento $(\mathrm{a} / \mathrm{c})$, a qualidade dos agregados e a porosidade (MEHTA; MONTEIRO, 2014).

Ainda de acordo com Mehta e Monteiro (2014), a resistência do concreto é a propriedade mais valorizada no controle de qualidade do compósito no estado endurecido. Pode ser definida como a capacidade de resistir às tensões sem o rompimento, sendo considerada pelo cálculo estrutural para dosagem do concreto e, após fabricado, para análise do controle de qualidade da mistura obtida.

Além da resistência à compressão, interessam outras propriedades, como a capacidade de resistência à tração (embora reduzida com relação àquela, em casos como pavimentos rígidos deve ser considerada). Essas duas propriedades serão descritas nos tópicos 2.1 e 2.2. Após os quais será comentado acerca do controle tecnológico para certificação do atendimento das propriedades requeridas em projeto e de pavimentos rígidos.

\subsection{Resistência à compressão simples}

A resistência à compressão simples $\left(f_{c}\right)$ é a propriedade mais importante do concreto, sendo sempre um dos parâmetros utilizados no dimensionamento da mistura. A maneira mais utilizada de verificar o atendimento a esse parâmetro é através da realização de ensaios de rompimento de corpos de prova (CP), moldados e curados de acordo com a ABNT (2015a), ensaiados conforme ABNT (2018), de acordo com a amostragem recomendada por ABNT (2015b), a idades de 28 dias ou outras $(j)$, desde que indicadas.

O ensaio é realizado através de um dispositivo que aplica os esforços de compressão, apresentando a carga máxima aplicada no momento imediatamente anterior ao rompimento do CP, o que torna possível obter as resistências à compressão individuais $\left(f_{c i}\right)$. Para determinação da resistência característica à compressão $\left(f_{c k}\right)$, são selecionados os maiores valores dos pares de CP utilizados, de $n$ conjuntos de amostragens. Com um valor de $n$ elevado, de acordo com o teorema do limite central (TLC), a distribuição dos dados de $f_{c}$ tende a seguir uma distribuição gaussiana (ou distribuição normal) Fig.1. 
Figura 1 - Curva de Gauss para a resistência do concreto à compressão.

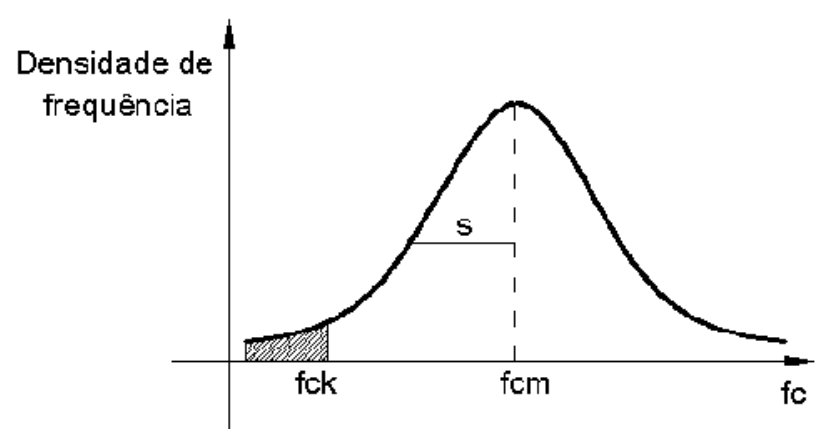

Fonte: Pinheiro, 2007.

Pode-se observar, pelo gráfico da Figura 1, que $f_{c k}$ é o valor da resistência à compressão que tem $5 \%$ de probabilidade de não ser alcançado em ensaios de CP de um determinado lote de concreto. O s é o desvio padrão e $f_{c m}$ é a resistência média desse concreto à compressão. Assim, $f_{c k}$ pode ser obtido de acordo com a Eq. (1), sendo o valor de 1,65 oriundo da tabela da distribuição normal padrão estatística para a de 0,05, e corresponde ao quantil de $5 \%$. Todos os valores são expressos em MPa.

$$
f_{c k}=f_{c m}-1,65^{*} s
$$

Equação (1)

\subsection{Resistência à tração}

A medida de resistência à tração do concreto pode ser tomada de três maneiras: uma forma direta e duas indiretas. A medida de tração direta pode ser efetuada através da fixação de dispositivo que aplicará esforços de tração ao CP Fig.2.

Figura 2 - Ensaio de tração direta.

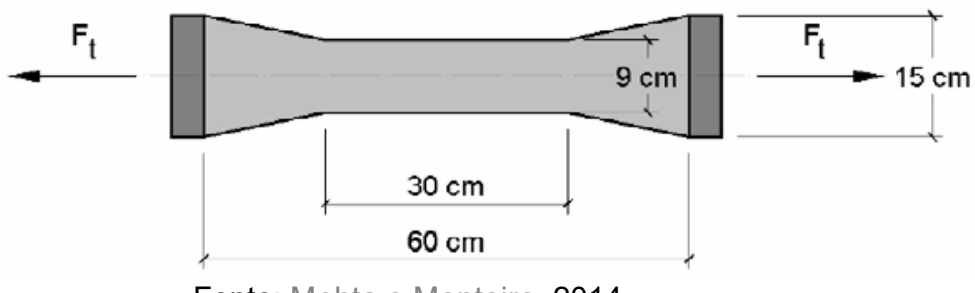

Fonte: Mehta e Monteiro, 2014.

Esse tipo de ensaio não é comum, já que, de acordo com Mehta e Monteiro (2014), os dispositivos que são fixados às extremidades do CP aplicam tensões secundárias, que não são mensuráveis podem interferir no resultado obtido. Assim, existem ensaios que são capazes de medir indiretamente a tração, que pode ser obtida através de relações matemáticas. Uma dessas medidas indiretas de resistência à tração é o ensaio de tração por compressão diametral Fig.3, conhecido mundialmente como "Brazilian Test". 
Figura 3 - Ensaio de tração por compressão diametral.

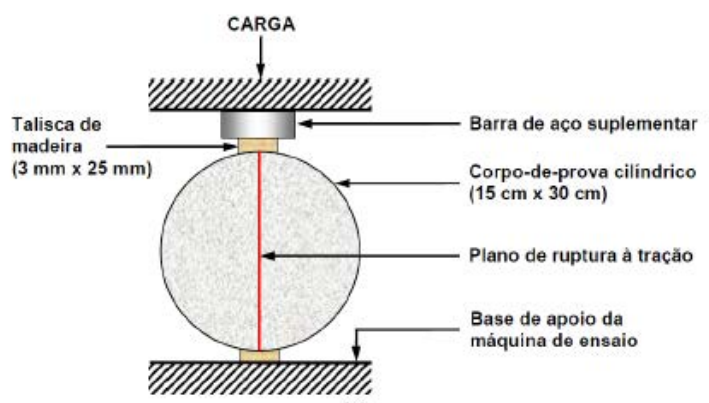

(a)

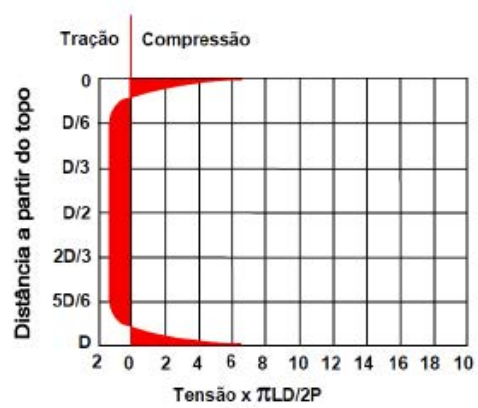

(b)

Fonte: Mehta e Monteiro, 2014.

A resistência à tração indireta por meio da compressão diametral $\left(f_{c t, s p}\right)$ e da resistência à tração na flexão $\left(f_{c t, f}\right)$ Fig.4 devem ser obtidas em ensaios realizados segundo as normas ABNT (2011) e ABNT (2010), respectivamente.

Figura 4 - Ensaio de tração na flexão.

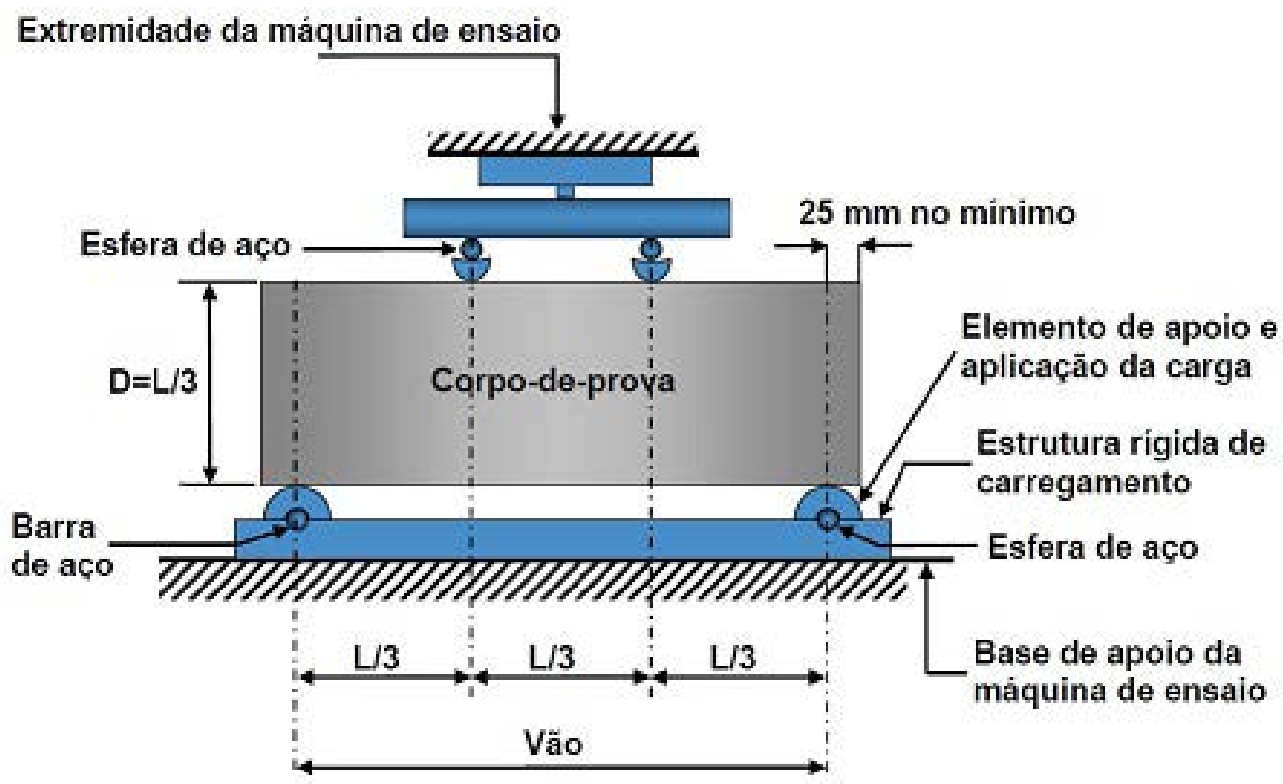

Fonte: Mehta e Monteiro, 2014.

A resistência à tração direta $\left(f_{c t}\right)$, por se tratar de um ensaio não realizado comumente, pode ser obtida dos demais ensaios. Caso o ensaio realizado tenha sido a compressão diametral, pode-se utilizar a Eq.(2) para cálculo de $f_{c t^{\prime}}$ Já para encontrar esse valor com base no resultado do ensaio de tração na flexão, emprega-se a Eq.(3). Essas duas equações são recomendadas por ABNT (2014).

$$
\begin{aligned}
f_{c t} & =0,9{ }^{*} f_{c t, s p} \\
f_{c t} & =0,7^{*} f_{c t, f}
\end{aligned}
$$$$
\text { Equação (2) }
$$ 
Os dados de resistência à tração podem ser analisados estatisticamente, de forma análoga ao apresentado no item 2.1. A resistência à tração direta média $\left(f_{c t m}\right)$, que é a média aritmética dos resultados, pode ser obtida com o valor encontrado de $f_{c k}$, como apresentado na Eq.(4).

$$
f_{c t m}=0,3 * f_{c k}^{2 / 3}
$$

Equação (4)

\subsection{Correlação entre compressão e tração}

A ABNT (2014) prevê a possibilidade de obtenção da resistência à tração com base no valor da resistência à compressão, ou vice-versa, conforme Eq. (4). De acordo com Chhorn et al. (2018), poucos estudos têm empregado esforços em analisar a relação entre os dois parâmetros. Em seu trabalho, esses autores buscaram analisar a relação entre a resistência à compressão e à tração de concreto compactado a rolo e comparar os resultados ao esperado para concreto convencional. Obtiveram a Eq.(5), da correlação entre a resistência à compressão e à tração na flexão, e a Eq.(6), da correlação entre a resistência à compressão e os dados obtidos da compressão diametral, com coeficientes de determinação $\left(R^{2}\right)$ de 0,59 e 0,62 , respectivamente.

$$
\begin{aligned}
& f_{c t, f}=0,678{ }^{*} f_{c}^{0,605} \\
& f_{c t, s p}=0,47^{*} f_{c}^{0,511}
\end{aligned}
$$$$
\text { Equação (6) }
$$

Os valores de $\mathrm{R}^{2}$ obtidos não são considerados representativos, já que menos de $70 \%$ dos valores de resistência à compressão explicam adequadamente os dados de resistência à tração. Outros autores buscaram realizar os estudos de correlação entre os parâmetros.

Já Pul (2008), por sua vez, obteve valores de $R^{2}$ que podem ser avaliados como mais relevantes. A resistência à compressão foi correlacionada com a compressão diametral na Eq.(7) e com a tração na Eq.(8), com coeficientes de determinação de 0,97 e 0,99, respectivamente.

$$
\begin{aligned}
& f_{c t, s p}=0,106{ }^{*} f_{c}^{0,948} \\
& f_{c t, f}=0,034{ }^{*} f_{c}^{1,286}
\end{aligned}
$$

Com os dados do segundo autor, pode-se notar que é possível a obtenção de $\mathrm{R}^{2}$ satisfatório para estimar os valores de resistência à tração com base nos dados obtidos de resistência à compressão axial.

\subsection{Pavimento}

Segundo DNIT (2006), os pavimentos são classificados, basicamente, em três categorias: flexíveis, também chamados de pavimentos asfálticos, rígidos, com nomenclatura de pavimentos de concreto e semirrígido (ou semiflexível).

De acordo com Cintra (2012), os pavimentos semirrígidos apresentam uma classificação intermediária, pois apresentam as duas funções primordiais expostas anteriormente: a de atuar sobre esforços de tração, porém simultaneamente, sem deixar de possuir as peculiaridades de um pavimento flexível. Isto ocorre porque a camada de base é executada com adição de algum aglomerante reativo, que vai estabilizar quimicamente a mistura, como solo-cimento, solo-cal e solo-betume.

Para o DNIT (2005), a seção transversal de um pavimento é composta pela fundação (subleito) e camadas sobrepostas com espessuras e materiais que atendam às necessidades exigidas, determinadas por meio de algum método de dimensionamento. Caso o pavimento seja flexível ou semirrígido, as camadas são, acima do subleito: reforço do subleito (se necessário), sub-base, base e revestimento, e a distribuição de cargas é mais localizada Fig.5. Caso o pavimento seja rígido, acima do subleito podem haver as camadas de sub-base, base e as placas de concreto, ou uma camada de sub-base seguida das placas rígidas, que podem assumir a dupla função de base e revestimento simultaneamente, e a distribuição de cargas é mais distribuída Fig.6. 
Figura 5 - Distribuição da carga sobre o pavimento flexível.

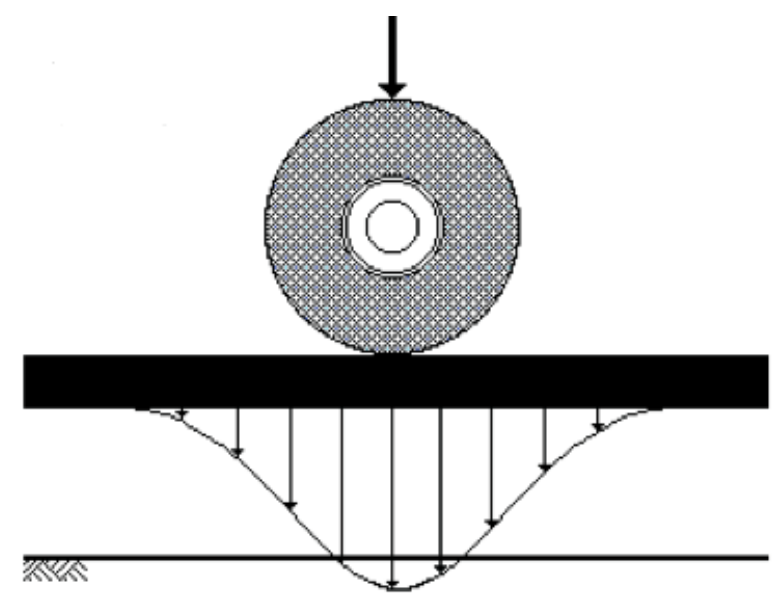

Fonte: Cintra, 2012.

Figura 6 - Distribuição da carga sobre o pavimento rígido.

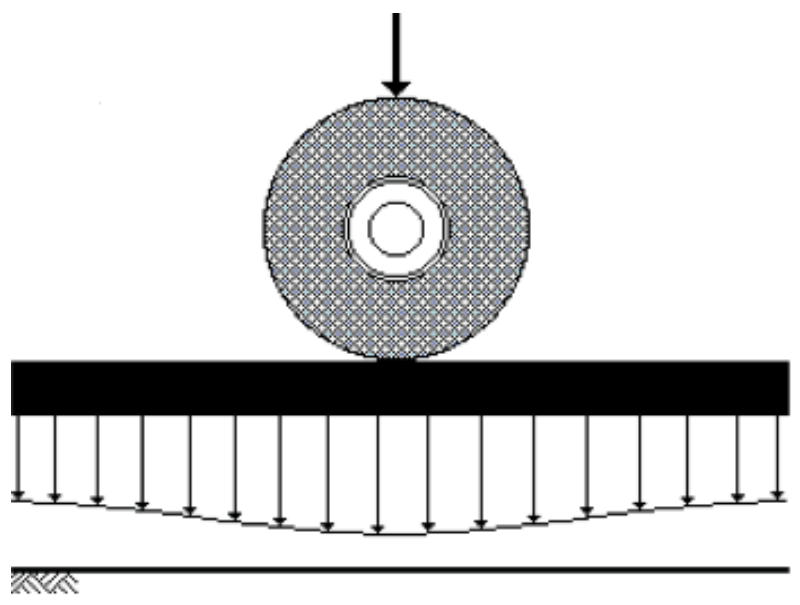

Fonte: Cintra, 2012.

Segundo Pitta (2000), em relação ao elevado valor do pavimento rígido, quando comparado ao do pavimento flexível, a diferença no custo inicial é compensada pela manutenção pesada ou reforço, que, no caso do concreto, só é necessária após 10 anos, enquanto que o asfalto necessita de reforço ou recapeamento a partir do $5^{\circ}$ ano.

De acordo com Bianchi et al. (2008), a principal diferença entre o pavimento rígido e o flexível é a forma como as cargas são distribuídas nas camadas subjacentes. De forma resumida, como citam Araújo et al. (2016), enquanto no pavimento rígido funciona em caráter estrutural, absorvendo a maior parte das tensões aplicadas, distribuindo-as de forma mais suave ao subleito, no flexível as cargas aplicadas no terreno natural são pontuais e de maior magnitude.

Pode-se observar, portanto, que as características do pavimento rígido têm a tendência de aliviar as tensões no subleito, pois a carga aplicada é mais distribuída no topo, diferentemente do que ocorre nos pavimentos flexíveis. Ressalta-se que o pavimento rígido pode não ser, necessariamente, a opção mais viável em todos os casos. Dado os custos de construção, a necessidade de disponibilidade de materiais específicos (como o cimento Portland e o aço das barras presentes nas placas), além da mão de obra mais qualificada, 
cada caso deve ser analisado em suas especificidades, e a solução de pavimentação a ser adotada deve ser a que apresenta a maior eficácia e melhor viabilidade técnico-econômica.

\section{Materiais e métodos}

\subsection{Caracterização da área de estudo}

Localizado na Rodovia BR-020/CE, o trecho analisado neste artigo consiste em $32 \mathrm{~km}$ de duplicação, sendo cerca de 26,1 km de pavimento rígido e $6 \mathrm{~km}$ de pavimento flexível Fig.7. A obra ainda se encontra em processo de execução. Foi iniciada em março de 2010, com previsão inicial de término em dezembro de 2015, porém foi paralisada e posteriormente retomada em 2018, com liberação ao tráfego em fevereiro de 2019 (CEARÁ, 2019). Realizada por um consórcio de construtoras com um valor contratual de cerca de $\mathrm{R} \$ 220,5$ milhões, está sob gestão da Superintendência de Obras Públicas do Governo do Estado do Ceará (SOP/CE), sob supervisão do Departamento Nacional de Infraestrutura de Transportes (DNIT).

Figura 7 - Trecho com pavimento já executado.

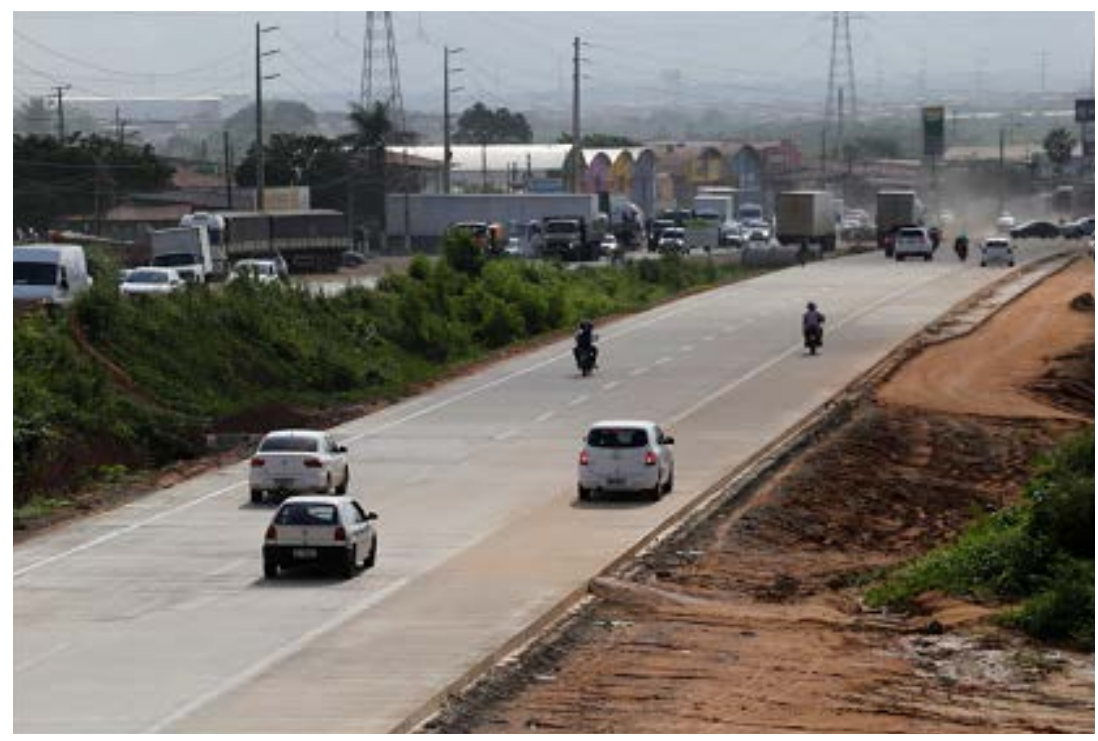

Fonte: Ceará, 2019.

É considerada uma obra de grande importância, já que possui como objetivo a construção e reconstrução da malha viária na área de influência de uma das mais importantes rodovias do Ceará. Tem como finalidade eliminar o congestionamento causado pelo grande número de veículos pesados que trafegam entre os municípios de Fortaleza e Região Metropolitana e de reduzir a incidência de acidentes na região.

O projeto contempla a implantação de uma pista Fig.8, em pavimento rígido, e a ampliação e restauração da existente Fig.9, em pavimento flexível. As duas pistas contemplam faixa de segurança, acostamento e ciclovia. Os projetos de execução foram elaborados de acordo com DNIT (2009). 
Figura 8 - Seção transversal da pista nova - Implantação (dimensões em metros; sem escala).

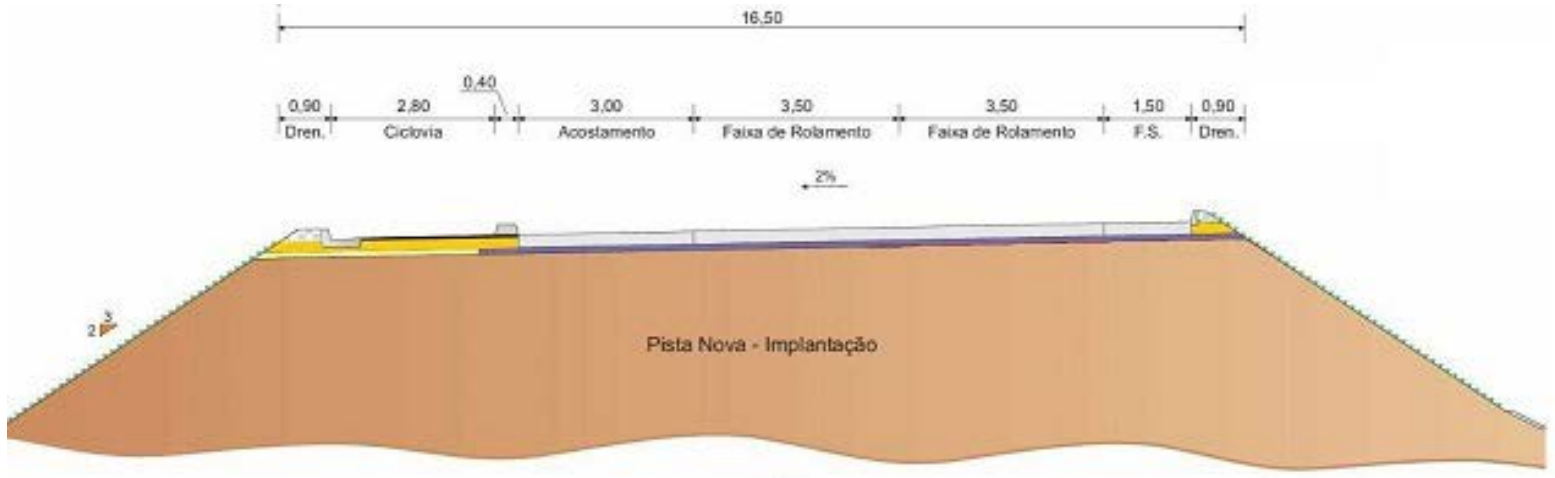

Fonte: DNIT, 2019.

Figura 9 - Seção transversal da pista existente (dimensões em metros; sem escala).

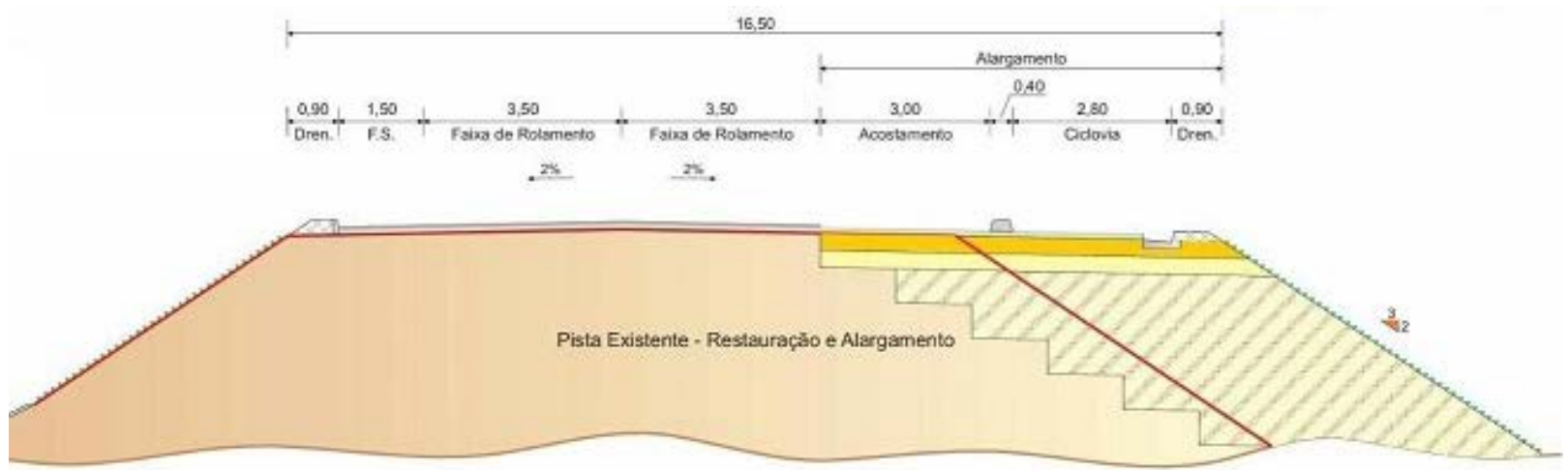

Fonte: DNIT, 2019.

A pista existente na Rodovia Quarto Anel Viário de Fortaleza foi executada em duas fases: a primeira em 1982 e a segunda em 1986. Já foram efetuadas diversas restaurações, sendo a última em 1992, quando foi executada uma camada de areia asfalto usinada a quente (AAUQ) e, no trecho referente à segunda fase, foi realizado um recapeamento da pista com concreto asfáltico usinado a quente (CAUQ). Atualmente, a pista e o acostamento possuem 7,0 m e 2,5 m de largura, respectivamente (BRITO, 2015).

Ainda de acordo com Brito (2015), na ampliação da pista existente e na construção da pista duplicada, foram adotadas soluções diferenciadas. A primeira foi implementada toda em pavimento flexível e a duplicação foi executada em PCS, com barras de transferência e de ligação, com exceção da ciclovia e da duplicação da pista entre a BR-222 e o município de Caucaia. Essas últimas foram executadas em pavimento flexível, possuindo uma camada de base em solo brita e outra de CAUQ, com espessura de $6,5 \mathrm{~cm}$ e uma camada de ligação de $6,0 \mathrm{~cm}$.

De acordo com Brito (2015), o novo pavimento de concreto da Rodovia Quarto Anel Viário está sendo executado com a seguinte estrutura:

- Sub-base de concreto compactado com rolo (CCR), espessura final de $15 \mathrm{~cm}$

- PCS de cimento Portland com inserção de barras de transferência e de ligação e espessura entre $22 \mathrm{~cm}$ e $24 \mathrm{~cm}$;

- Concreto com traço unitário em massa de materiais secos com a ordem: cimento Portland (CP II F 32), areia, brita $19 \mathrm{~mm}$, brita $32 \mathrm{~mm}$, água/cimento, aditivo plastificante $(1: 1,82: 1,52: 1,36: 0,40$ : 0,60), conforme DNIT (2004); 
- Resistência à compressão axial igual ou superior a $30 \mathrm{MPa}$, aos 28 dias de idade;

- Resistência à tração na flexão igual ou superior a 4,5 MPa, aos 28 dias de idade;

- Abatimento de tronco de cone de $30 \mathrm{~mm} \pm 10 \mathrm{~mm}$;

- Início da pega em 30 min, com cura química à base de hidrocarboneto parafínico.

\subsection{Coleta e análise de dados}

Para análise da correlação entre os dados de resistência mecânica foram coletadas as informações oriundas do controle tecnológico. A empresa responsável moldou 8 CP para realização dos ensaios de compressão axial simples (cilíndricos, com dimensões de $10 \mathrm{~cm}$ de diâmetro por $20 \mathrm{~cm}$ de altura) e tração na flexão (prismáticos, com seção transversal quadrada medindo $15 \mathrm{~cm}$ e comprimento de $50 \mathrm{~cm}$ ), aos 7 e 28 dias, a cada descarregamento do caminhão betoneira, e a cada $50 \mathrm{~m}$ de concretagem de placa.

A forma de repasse dos dados para este artigo foi realizada em três planilhas diferentes, com todas as informações obtidas. Esses três conjuntos foram mantidos, identificados sequencialmente como 1, 2 e 3, e a idade de controle considerada dos dados de resistência mecânica foi de 28 dias. A Tabela 1 apresenta um resumo dos dados coletados na pista esquerda, que é a pista correspondente ao pavimento rígido implantado.

Tabela 1 - Dados coletados dos ensaios de controle tecnológico do concreto.

\begin{tabular}{c|c|c}
\hline \multirow{2}{*}{ Identificação } & \multicolumn{2}{|c}{ Quantidade de pares de CP } \\
\cline { 2 - 3 } & Tração na flexão & Compressão \\
\hline 1 & 37 & 37 \\
\hline 2 & 38 & 38 \\
\hline 3 & 49 & 49 \\
\hline Total & 124 & 124 \\
\hline
\end{tabular}

Fonte: Os autores, 2019.

Para cada um dos três conjuntos de dados, foram calculados $f_{c m}, f_{c k}, f_{c t m}$ com base no $f_{c t, f}, f_{c t, f}$ média e desvio padrão (s). Essas informações foram utilizadas para o cálculo da correlação entre o $f_{c k}$ e o $f_{c t, f}$ Para tanto, foram consideradas as equações (3) e (4), para calcular a variável independente $\left(f_{c}\right)$ e a dependente $\left(f_{c t, f}\right)$.

Inicialmente, as equações (3) e (4) foram relacionadas. Após essa etapa, isolou-se o termo referente à variável dependente Eq 9. Como se deve utilizar uma equação de reta para análise com uso de regressão linear simples, a obtida foi linearizada com uso de logaritmo na base 10, obtendo-se a Equação (10).

$$
\begin{array}{cr}
f_{c t, f}=0,43 * f_{c k}^{2 / 3} & \text { Equação (9) } \\
\log _{10} f_{c t, f}=\log _{10} 0,43+2 / 3 * \log _{10} f_{c k} & \text { Equação (10) }
\end{array}
$$

Analogamente à equação da reta, tem-se que $y=\log _{10} f_{c t, f}$ e $x=\log _{10} f_{c k}$. Foram, então, criadas colunas com os cálculos de $x$ e $y$ para efetuar a análise de regressão linear simples com uso do método dos mínimos quadrados (MMQ). Os dados de resistência à compressão e à tração na flexão utilizados são os valores máximos de cada par, como indicado na ABNT (2015b). De posse dessas informações, tem-se uma equação que correlaciona os dados analisados.

Para se ter ideia da eficiência da Eq.(10), os dados de $f_{c t, f}$ foram calculados com base no $f_{c k}$ obtido nos ensaios para verificação do erro que essa equação é capaz de gerar. Comparativamente, foi utilizada a Equação (9), que leva em consideração as recomendações estabelecidas na ABNT (2014). A forma mais adequada de obter os dados de tração é aquela que obtém o menor erro. O cálculo de erro utilizado neste trabalho foi a raiz do erro quadrático médio (Root Mean Square Error - RMSE), conforme Eq.(11).

$$
R M S E=\sqrt{\frac{\sum_{i}^{n}\left(O_{i}-P_{i}\right)^{2}}{n}}
$$


Em que, $O_{i}$ são os valores observados;

$P_{i}$ são os valores previstos (ou calculados), e

$n$ é a quantidade de dados da amostra analisada.

O resultado obtido da aplicação da Eq.(11) tem a mesma unidade que os dados observados e calculados, que, no caso deste trabalho, é a unidade de resistência mecânica, em MPa.

\section{Resultados}

A Tabela 2 apresenta um resumo descritivo dos dados obtidos do controle tecnológico na fabricação das placas de concreto do PCS analisado.

Tabela 2 - Resumo descritivo dos dados de concreto (em MPa).

\begin{tabular}{|c|c|c|c|c|}
\hline \multirow{2}{*}{ Ensaio } & \multirow{2}{*}{ Variáveis } & \multicolumn{3}{|c|}{ Conjuntos de dados } \\
\hline & & 1 & 2 & 3 \\
\hline \multirow{7}{*}{ Compressão } & $f \mathrm{~cm}$ & 38,34 & 39,49 & 43,00 \\
\hline & $f \mathrm{ck}$ & 35,04 & 36,25 & 39,70 \\
\hline & Erro Padrão & 0,31 & 0,32 & 0,28 \\
\hline & $s(f \mathrm{C})$ & 1,92 & 1,96 & 1,94 \\
\hline & $c v(f \mathrm{c})$ & 0,05 & 0,05 & 0,04 \\
\hline & Máx. & 45,5 & 44,4 & 48,0 \\
\hline & Mín. & 34,3 & 36,0 & 38,0 \\
\hline \multirow{6}{*}{ Tração na flexão } & $f c t, f$, méd & 5,55 & 5,75 & 6,02 \\
\hline & Erro Padrão & 0,09 & 0,06 & 0,06 \\
\hline & $s(f c t)$ & 0,58 & 0,39 & 0,43 \\
\hline & $c v(f \mathrm{ct})$ & 0,10 & 0,07 & 0,07 \\
\hline & Máx. & 6,60 & 6,60 & 6,70 \\
\hline & Mín. & 4,50 & 5,00 & 5,20 \\
\hline
\end{tabular}

Fonte: Os autores (2019).

Uma das considerações que pode ser realizada com esses resultados é com relação à aceitação dos valores encontrados. Todos os valores de $f_{c k}$ foram superiores a $30 \mathrm{MPa}$, e os de $f_{c t, f \text { méd }}$ foram superiores a 4,5 MPa, fazendo com que essas placas sejam aprovadas com relação à resistência mecânica mínima para concreto utilizado em pavimentação. Uma avaliação a ser realizada é com relação ao coeficiente de variação (cv).

Em todos os casos, de compressão e tração na flexão dos três conjuntos amostrais, os valores de desvio padrão $(s)$ e o coeficiente de variação $(c v)$ foram baixos, o que pode levar à confirmação de dados pouco 
dispersos. Os gráficos das figuras 10, 11 e 12 apresentam a dispersão dos três conjuntos amostrais utilizados neste trabalho (apresentados no anexo).

Figura 10 - Dispersão dos dados do conjunto amostral 1.

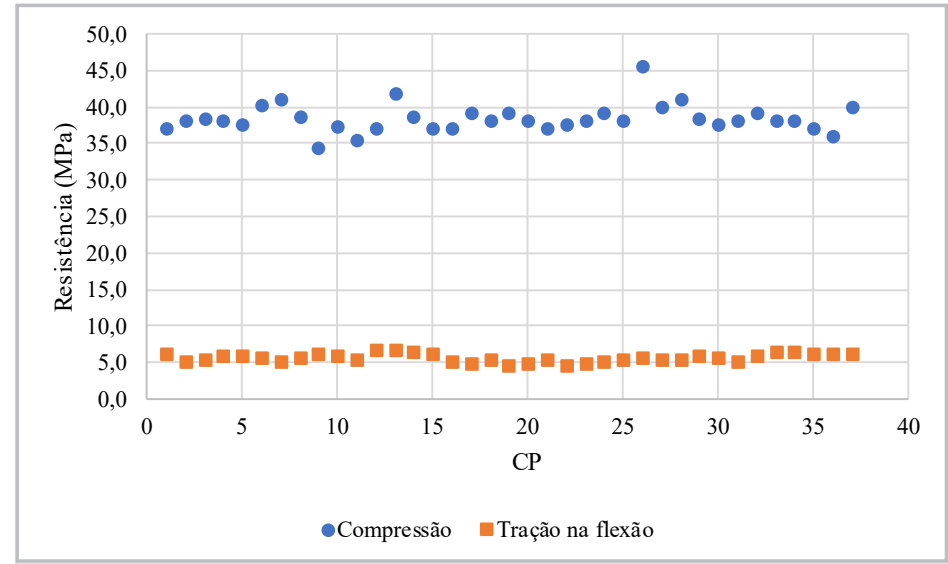

Fonte: Os autores, 2019.

Figura 11 - Dispersão dos dados do conjunto amostral 2.

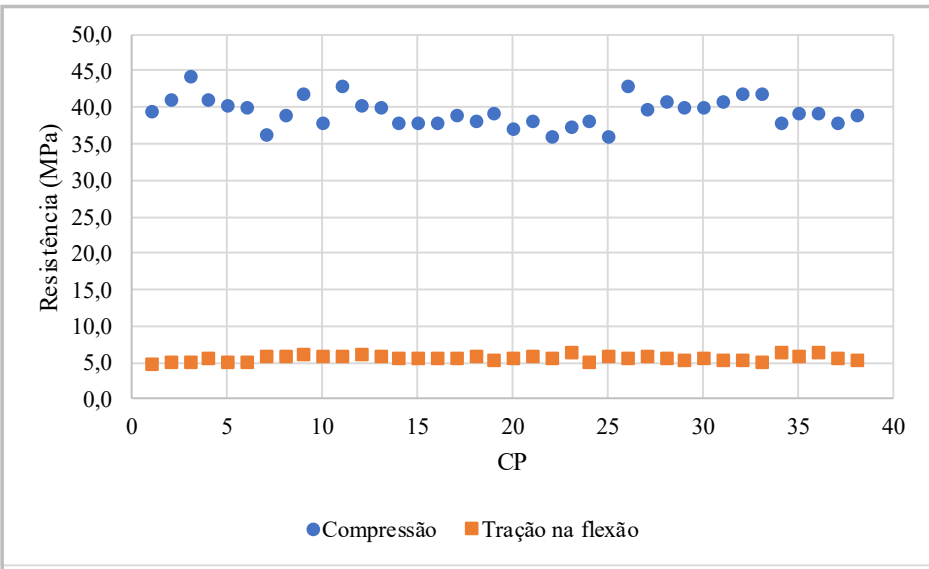

Fonte: Os autores, 2019.

Figura 12 - Dispersão dos dados do conjunto amostral 3.

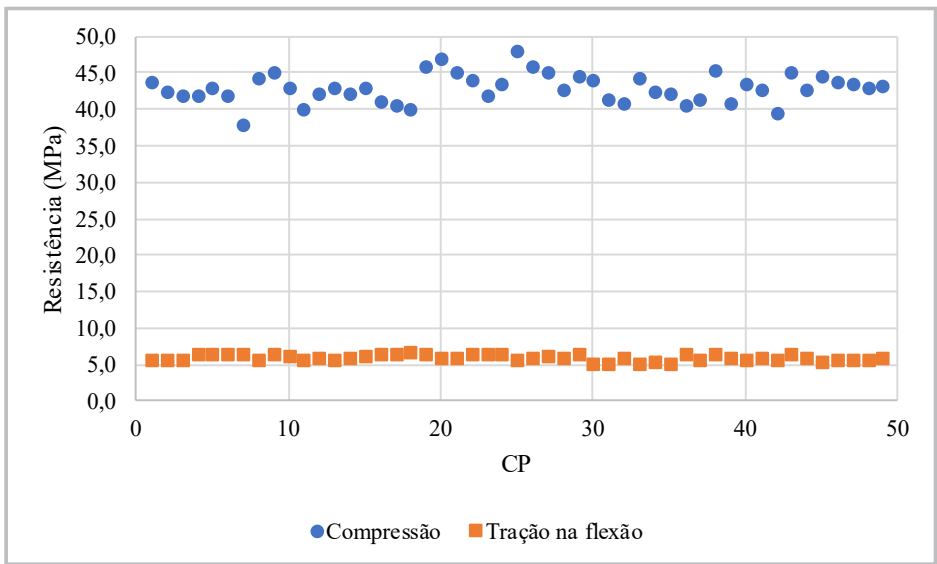

Fonte: Os autores, 2019. 
Como se pode observar nos gráficos, os dados não apresentam dispersão elevada, como esperado com o resultado do $c v$. Para o caso do desvio padrão calculado, os valores dos conjuntos amostrais 1, 2 e 3, respectivamente, foram de 1,92, 1,96 e 1,94, que podem ser considerados valores próximos. Porém, para o cálculo do $f_{c k}$ com o uso da Eq.(1), foi adotado o valor mínimo do desvio padrão indicado por ABNT (2015b), de 2,0 MPa. A Tabela 3 apresenta os dados calculados para a regressão linear simples, conforme Eq.(10).

Tabela 3 - Dados para regressão linear simples (em MPa).

\begin{tabular}{c|c|c|c|c}
\hline ID & $\begin{array}{c}\boldsymbol{f}_{c k} \\
(\mathbf{A})\end{array}$ & $\boldsymbol{f}_{c t, f, \boldsymbol{m e ́ d}}(\mathbf{B})$ & $\begin{array}{c}\boldsymbol{x} \\
\log _{10}(\boldsymbol{A})\end{array}$ & $\begin{array}{c}\boldsymbol{y} \\
\log _{10}(\mathbf{B})\end{array}$ \\
\hline 1 & 35,04 & 5,6 & 1,545 & 0,748 \\
\hline 2 & 36,25 & 5,8 & 1,559 & 0,763 \\
\hline 3 & 39,70 & 6,0 & 1,599 & 0,778 \\
\hline
\end{tabular}

Fonte: Os autores, 2019.

Os parâmetros $x$ e $y$ são, respectivamente, as variáveis independente e dependente a serem calculadas. A Figura 13 apresenta o gráfico obtido da regressão linear simples utilizando o método dos mínimos quadrados (MMQ) com a equação da reta de ajuste dos dados, e o valor do coeficiente de determinação $\left(R^{2}\right)$.

Figura 13 - Reta obtida através do MMQ.

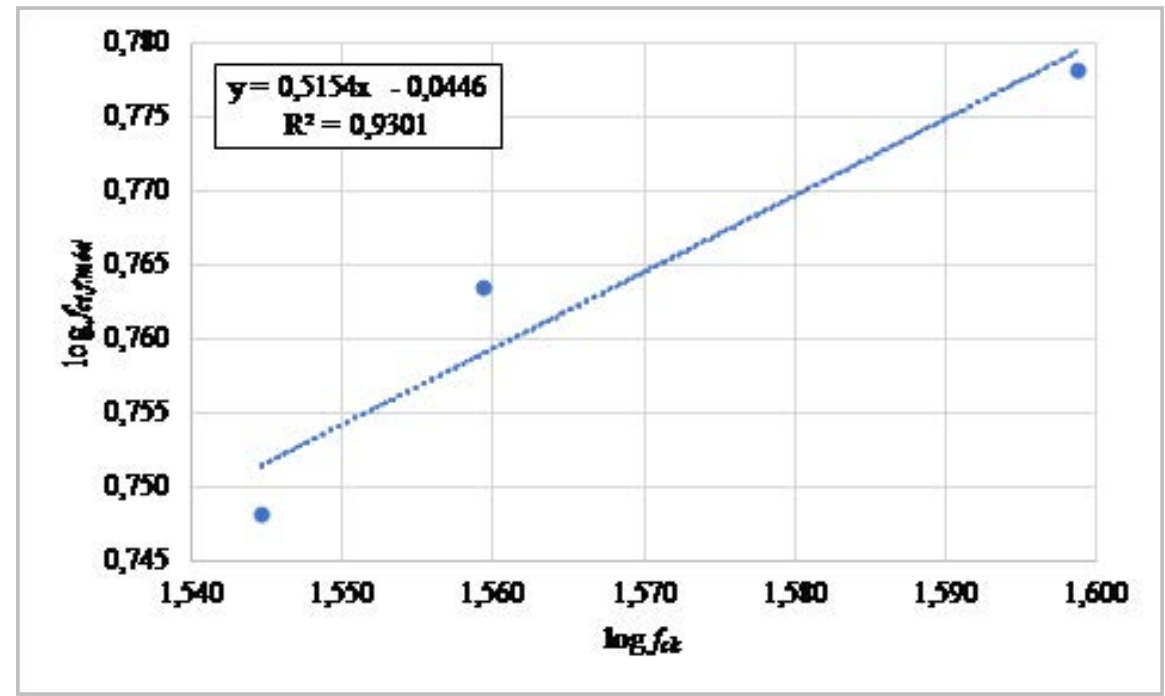

Fonte: Os autores, 2019.

A equação encontrada pelo $\mathrm{MMQ}$, que correlaciona os dados, resultou em um coeficiente de determinação $\left(R^{2}\right)$ de 0,9301 , valor que pode ser considerado como bom, pois significa que $93,01 \%$ dos dados dependentes podem ser explicados pela variável independente. Logo, com essa equação da reta de ajuste, e sabendo que $y=\log _{10} f_{c t, f}$ e $x=\log _{10} f_{c k}$, obtém-se a Eq.(12) que correlaciona os dados, analogamente àquela apresentada por ABNT (2014).

$$
f_{c t, f}=0,902 * f_{c k}^{0,52}
$$

Como a Equação (12) difere da Equação (9), foram calculados os dados de $f_{c t, f}$ com ambos, e verificados os erros oriundos dessas estimativas. A Tabela 4 apresenta os resultados dos erros calculados, com uma resistência à tração na flexão média de $5,8 \mathrm{MPa}$, considerando os três conjuntos amostrais. 
Tabela 4 - Dados do RMSE (em MPa).

\begin{tabular}{c|c|c|c}
\hline \multirow{2}{*}{ ID } & \multirow{2}{*}{$\boldsymbol{f}_{\text {ct,f,méd }}$} & \multicolumn{2}{|c}{$\boldsymbol{R M S E}$} \\
\cline { 3 - 4 } & 5,6 & Equação (5) & Equação (8) \\
\hline 1 & 5,8 & 0,90 & 0,80 \\
\hline 2 & 6,0 & 0,90 & 0,60 \\
\hline 3 & 5,8 & 0,90 & 0,60 \\
\hline Média & 0,90 & 0,67 \\
\hline
\end{tabular}

Fonte: Os autores, 2019.

Ao considerar os erros obtidos da Equação (9), da ABNT (2014), o RMSE médio foi de 0,90, enquanto que o erro calculado dos dados obtidos com a aplicação da Eq.(8), encontrada com os dados analisados, foi de 0,67 . Estes são valores consideravelmente baixos, o que, devido à proximidade, indica que a equação proposta pela ABNT (2014) pode ser aplicada.

Já para os dados médios da resistência à tração na flexão, com o erro encontrado, todos os casos ainda levariam o concreto utilizado na confecção das placas à aceitação, pois atingem valores acima do mínimo estabelecido de 4,5 MPa (o mínimo encontrado no controle tecnológico foi de 4,7 MPa). Assim, ao empregar a Equação (9), pode existir uma economia com relação aos ensaios de controle tecnológico, já que é mais simples efetuar ensaios de resistência à compressão simples, com menor consumo de material para tal atividade.

\section{Conclusões}

Pavimentos rígidos com emprego de concreto de cimento Portland são uma alternativa viável para trechos com alto volume de tráfego e veículos comerciais pesados, como é o caso da Rodovia Quarto Anel Viário de Fortaleza. Como esse tipo de obra demanda alto volume de concreto, e o mesmo tem uma área superficial elevada que está em contato com o ambiente, desfavorecendo seu processo de cura, é de extrema importância que sejam realizados ensaios mecânicos para controle tecnológico da produção.

Para o caso de pavimento de concreto simples, em que não há necessidade de armadura, há uma exigência quanto à resistência à tração na flexão, que deve atingir, no mínimo, 4,5 MPa na idade de controle. Quando há necessidade de armaduras, também há uma exigência mínima de resistência à compressão, no valor de $30 \mathrm{MPa}$. No caso de estudo deste artigo, foi realizado controle tecnológico dos dois parâmetros de resistência mecânica: à compressão e à tração na flexão.

Os dados obtidos foram tratados e analisados através de regressão linear simples, com o uso do método dos mínimos quadrados. Obteve-se, ao final, uma equação que difere da prevista na norma em questão. Para avaliar a acurácia dos resultados da estimativa indireta da resistência à tração na flexão, utilizaram-se a relação encontrada e a normalizada. Esse passo serviu para calcular, com a referência dos dados reais, o erro que cada equação gera e verificar qual seria a equação viável.

Os erros calculados da aplicação das duas equações foram muito próximos, e podem ser considerados similares, porém o menor valor foi o obtido com a equação encontrada neste estudo. Embora a equação tenha sido distinta, para os casos em que não é possível obter uma das variáveis por meio de ensaios com corpos de prova, pode-se estimar a outra variável com base na equação normalizada. Essa diferença pode ocorrer devido aos fatores que interferem no ganho de resistência mecânica do concreto, tais como: teor de argamassa, qualidade dos agregados utilizados, dosagem, mistura, transporte, lançamento, adensamento, processo de cura, teor de ar incorporado e o fator água/cimento.

Vale salientar que a equação aqui encontrada se refere somente aos dados deste artigo. Já a equação normalizada pode ter aplicação em qualquer conjunto de dados. Assim, dados os erros encontrados, podese utilizar a equação da norma. Caso seja possível a obra obter sua própria equação, a precisão dos dados obtidos, considerando as condições similares dos concretos produzidos, seria ampliada. 


\section{Referências}

ARAÚJO, M. A. et al. Análise comparativa de métodos de pavimentação - pavimento rígido (concreto) $x$ flexível (asfalto). Revista Científica Multidisciplinar Núcleo do Conhecimento, São Paulo, ano 1, ed. 11, v. 10, p. 187-196, nov. 2016.

ASSOCIAÇÃO BRASILEIRA DE NORMAS TÉCNICAS. NBR 6118: Projeto de estruturas de concreto Procedimento. Rio de Janeiro: ABNT, 2014.

ASSOCIAÇÃO BRASILEIRA DE NORMAS TÉCNICAS. NBR 5738: Concreto - Procedimento para moldagem e cura de corpos de prova. Rio de Janeiro: ABNT, 2015a.

ASSOCIAÇÃO BRASILEIRA DE NORMAS TÉCNICAS. NBR 12655: Concreto de cimento Portland Preparo, controle, recebimento e aceitação - Procedimento. Rio de Janeiro: ABNT, 2015b.

ASSOCIAÇÃO BRASILEIRA DE NORMAS TÉCNICAS. NBR 5739: Concreto - Ensaios de compressão de corpos-de-prova cilíndricos. Rio de Janeiro: ABNT, 2018.

ASSOCIAÇÃO BRASILEIRA DE NORMAS TÉCNICAS. NBR 7222: Concreto e argamassa - Determinação da resistência à tração por compressão diametral de corpos de prova cilíndricos. Rio de Janeiro: ABNT, 2011.

ASSOCIAÇÃO BRASILEIRA DE NORMAS TÉCNICAS. NBR 12142: Concreto - Determinação da resistência à tração na flexão de corpos de prova prismáticos. Rio de Janeiro: ABNT, 2010.

BIANCHI, F. R.; BRITTO, I. R. T.; CASTRO, V. A. B. Estudo comparativo entre pavimento rígido e flexível. In: CONGRESSO BRASILEIRO DE CONCRETO, 50., Salvador, 2008. Anais [...]. Disponível em: http:// www.ibracon.org.br/eventos/50cbc/pav_apresentacoes/ISIS_RAQUEL.pdf . Acesso em: 20 jun. 2019.

BRITO, L. R. Correlação entre a resistência à tração na flexão e a resistência à compressão axial em pavimentos rígidos. 2015. 69 f. TCC (Graduação em Engenharia Civil) - Universidade de Fortaleza, Fortaleza, 2015.

CEARÁ. Governo do Estado. $4^{\circ}$ Anel Viário: 32 quilômetros de pista duplicada são liberados - Notícia. Disponível em: https://www.ceara.gov.br/2019/02/19/4anel-viario-32-quilometros-de-pista-duplicada-saoliberados/. Acesso em: 15 maio 2019.

CHHORN, C.; HONG, S. J.; LEE, S.W. Relationship between compressive and tensile strengths of rollercompacted concrete. Journal of Traffic and Transportation Engineering, China, v. 5, n. 3, p. 215-223, jun. 2018.

CINTRA, P.G. Aplicação de resíduos de construção e demolição em estruturas de pavimento. 2012. 65 f. TCC (Graduação em Engenharia Civil) - Universidade Estadual Paulista Júlio de Mesquita Filho, Guaratinguetá, 2012.

CONFEDERAÇÃO NACIONAL DO TRANSPORTE. Transporte rodoviário: por que os pavimentos das rodovias do Brasil não duram? - Brasília: CNT, 2017.

DEPARTAMENTO NACIONAL DE INFRAESTRUTURA DE TRANSPORTES. Manual de pavimentação. 3. ed. Rio de Janeiro: DNIT, 2006. (IPR. Publ. 719).

DEPARTAMENTO NACIONAL DE INFRAESTRUTURA DE TRANSPORTES. Manual de pavimentos rígidos. 2. ed. Rio de Janeiro: DNIT, 2005. (IPR. Publ. 714).

DEPARTAMENTO NACIONAL DE INFRAESTRUTURA DE TRANSPORTES. Multimodalidade na Mobilidade [palestra]. In: ENCONTRO DE QUALIDADE E TECNOLOGIA DO TRANSPORTE URBANO - 
EMPRESA DE TRANSPORTE URBANO DE FORTALEZA, 4., 2009, Fortaleza. Anais [...]. Disponível em: http://www.etufor.ce.gov.br/pdfs/multimodalidade_mobilidade.pdf. Acesso em: 02 maio 2019.

DEPARTAMENTO NACIONAL DE INFRAESTRUTURA DE TRANSPORTES. Norma 054/2004-PRO:

Pavimento rígido - Estudo de traços de concreto e ensaios de caracterização de matérias - Procedimento.

Rio de Janeiro: DNIT, 2004.

MEHTA, P. K.; MONTEIRO, P. J. M. Concreto - estrutura, propriedades e materiais. 2. ed. São Paulo: IBRACON, 2014.

PINHEIRO, L. M. Fundamentos do concreto e projeto de edifícios. São Carlos: Universidade de São Paulo, 2007.

PITTA, M. R. Pavimento. Revista Téchne, São Paulo, 2000. Disponível em: http://techne.pini.com.br/ engenharia-civil/46/artigo285596-1.aspx. Acesso em: 09 maio 2019.

PUL, S. Experimental investigation of tensile behavior of high strength concrete. Indian Journal of Engineering \& Materials Sciences, Índia, v. 15, p. 467-472, 2008.

\section{Sobre os autores}

Luciana Barreto de Brito

Arquiteta e urbanista e Engenheira Civil pelo Centro de Ciências Tecnológicas da Universidade de Fortaleza (Unifor). Atualmente, é Engenheira Civil da Secretaria das Cidades do Governo do Estado do Ceará.

\section{Antonia Fabiana Marques Almeida}

Doutoranda em Engenharia de Transportes na Universidade Federal do Ceará (UFC). Mestre em Engenharia de Transportes pela UFC (2010). Graduada em Engenharia Civil pela UFC (2014). Tecnóloga em Estradas pelo Instituto Federal de Ciência e Tecnologia do Ceará (IFCE - 2005). Professora da Universidade Federal do Cariri (UFCA).

\section{Francisco Heber Lacerda de Oliveira}

Doutor em Engenharia de Transportes pela Universidade Federal do Ceará (UFC). Professor adjunto do Departamento de Engenharia de Transportes da UFC. Affiliate Member in the American Society of Civil Engineers (ASCE). Tem experiência em Planejamento do Transporte Aéreo; e Operação, Manutenção e Reabilitação de Infraestruturas Aeroportuárias, especialmente em pavimentos de pátios e de pistas de pouso e decolagem.

Recebido em: 05/08/2019

Aceito em: 27/09/2019 
ANEXO: Dados de resistência à compressão $\left(f_{c}\right)$ e à tração na flexão $\left(f_{t}\right)$ dos três conjuntos amostrais utilizados no estudo.

\begin{tabular}{|c|c|c|c|c|c|c|c|c|c|}
\hline Amostra & $f_{c 1}$ & $\mathbf{f}_{\mathrm{c} 2}$ & $f_{t 1}$ & $f_{12}$ & Amostra & $f_{c 1}$ & $\mathbf{f}_{\mathrm{c} 2}$ & $\mathbf{f}_{t 1}$ & $f_{t 2}$ \\
\hline 1 & 37,0 & 36,1 & 6,0 & 6,0 & 2 & 42,1 & 43,0 & 5,8 & 5,8 \\
\hline 1 & 35,0 & 38,0 & 5,1 & 4,5 & 2 & 39,9 & 38,0 & 5,8 & 6,1 \\
\hline 1 & 37,0 & 38,2 & 4,5 & 5,2 & 2 & 40,8 & 37,0 & 5,6 & 5,6 \\
\hline 1 & 37,0 & 38,0 & 5,5 & 5,7 & 2 & 40,0 & 40,1 & 5,5 & 5,4 \\
\hline 1 & 37,5 & 37,1 & 5,8 & 5,1 & 2 & 39,0 & 40,0 & 5,5 & 5,6 \\
\hline 1 & 40,1 & 39,0 & 5,5 & 5,6 & 2 & 39,3 & 40,9 & 5,4 & 5,5 \\
\hline 1 & 39,0 & 40,9 & 4,6 & 5,0 & 2 & 41,9 & 40,0 & 5,3 & 5,4 \\
\hline 1 & 38,1 & 38,7 & 5,1 & 5,5 & 2 & 41,6 & 42,0 & 5,3 & 5,2 \\
\hline 1 & 34,3 & 33,0 & 5,9 & 6,2 & 2 & 38,0 & 38,0 & 5,9 & 6,4 \\
\hline 1 & 37,3 & 36,0 & 5,9 & 5,6 & 2 & 39,1 & 39,3 & 6,1 & 5,0 \\
\hline 1 & 35,4 & 34,0 & 5,2 & 4,8 & 2 & 39,2 & 39,3 & 6,4 & 5,0 \\
\hline 1 & 36,9 & 35,1 & 6,6 & 6,4 & 2 & 38,0 & 38,0 & 5,1 & 5,8 \\
\hline 1 & 41,8 & 39,0 & 6,5 & 6,2 & 2 & 36,3 & 39,0 & 5,4 & 5,3 \\
\hline 1 & 38,4 & 38,5 & 5,5 & 6,3 & 3 & 43,7 & 39,4 & 5,3 & 5,8 \\
\hline 1 & 36,8 & 37,0 & 6,2 & 6,0 & 3 & 42,5 & 39,7 & 5,8 & 5,1 \\
\hline 1 & 36,1 & 37,0 & 5,0 & 5,0 & 3 & 39,7 & 42,0 & 5,2 & 5,8 \\
\hline 1 & 38,1 & 39,0 & 4,5 & 4,7 & 3 & 39,9 & 42,0 & 6,3 & 6,4 \\
\hline 1 & 38,0 & 37,3 & 5,2 & 5,0 & 3 & 43,1 & 43,0 & 6,5 & 6,3 \\
\hline 1 & 39,0 & 36,1 & 4,3 & 4,5 & 3 & 42,0 & 42,0 & 6,4 & 6,0 \\
\hline 1 & 38,0 & 38,0 & 4,0 & 4,8 & 3 & 38,0 & 37,6 & 5,7 & 6,5 \\
\hline 1 & 37,1 & 36,1 & 5,3 & 5,0 & 3 & 42,1 & 44,4 & 5,6 & 5,7 \\
\hline 1 & 37,2 & 37,4 & 4,2 & 4,6 & 3 & 43,6 & 45,0 & 6,4 & 6,5 \\
\hline 1 & 38,1 & 36,6 & 4,6 & 4,8 & 3 & 39,8 & 43,0 & 5,6 & 6,3 \\
\hline 1 & 38,0 & 39,0 & 5,1 & 5,0 & 3 & 39,8 & 40,1 & 5,0 & 5,6 \\
\hline 1 & 37,1 & 38,0 & 4,3 & 5,2 & 3 & 42,1 & 41,3 & 6,0 & 6,0 \\
\hline 1 & 45,5 & 43,1 & 5,5 & 5,6 & 3 & 40,6 & 43,0 & 5,4 & 5,6 \\
\hline 1 & 37,0 & 40,0 & 5,3 & 5,4 & 3 & 42,1 & 40,1 & 5,9 & 5,7 \\
\hline 1 & 40,9 & 39,1 & 5,2 & 5,2 & 3 & 43,0 & 38,0 & 5,7 & 6,3 \\
\hline 1 & 38,1 & 38,4 & 5,7 & 5,6 & 3 & 40,0 & 41,0 & 6,4 & 6,6 \\
\hline 1 & 37,3 & 37,4 & 5,6 & 5,6 & 3 & 40,7 & 40,3 & 5,9 & 6,5 \\
\hline 1 & 38,1 & 38,0 & 5,1 & 5,1 & 3 & 40,0 & 40,1 & 6,7 & 6,0 \\
\hline 1 & 39,0 & 37,0 & 5,9 & 5,3 & 3 & 46,0 & 41,1 & 6,5 & 6,3 \\
\hline 1 & 38,0 & 36,0 & 6,4 & 6 & 3 & 47,1 & 46,0 & 5,9 & 6,0 \\
\hline 1 & 37,5 & 38,0 & 6,3 & 6,1 & 3 & 45,0 & 40,0 & 6,1 & 6,0 \\
\hline 1 & 36,0 & 37,0 & 6,2 & 6 & 3 & 44,1 & 40,3 & 5,8 & 6,6 \\
\hline 1 & 34,2 & 36,0 & 5,8 & 6 & 3 & 42,0 & 38,0 & 6,4 & 5,7 \\
\hline 1 & 37,0 & 40,0 & 6,1 & 6 & 3 & 43,3 & 43,6 & 6,4 & 5,9 \\
\hline 2 & 39,6 & 35,2 & 5,0 & 5,0 & 3 & 44,6 & 48,0 & 5,8 & 5,7 \\
\hline 2 & 36,0 & 41,1 & 5,1 & 4,7 & 3 & 43,8 & 46,0 & 6,1 & 6,0 \\
\hline 2 & 42,2 & 44,4 & 4,6 & 5,3 & 3 & 44,8 & 45,0 & 6,3 & 6,2 \\
\hline 2 & 41,0 & 40,0 & 5,4 & 5,7 & 3 & 42,7 & 42,2 & 6,0 & 6,0 \\
\hline 2 & 37,1 & 40,3 & 4,1 & 5,3 & 3 & 44,0 & 44,5 & 6,4 & 5,9 \\
\hline 2 & 39,0 & 40,1 & 4,5 & 5,3 & 3 & 43,0 & 44,0 & 5,0 & 5,3 \\
\hline 2 & 36,2 & 36,4 & 6,0 & 5,8 & 3 & 41,5 & 41,2 & 5,3 & 5,2 \\
\hline 2 & 38,0 & 39,1 & 5,7 & 6,0 & 3 & 40,9 & 38,0 & 5,8 & 6,0 \\
\hline 2 & 41,8 & 38,0 & 5,8 & 6,3 & 3 & 44,0 & 44,2 & 5,2 & 5,1 \\
\hline 2 & 37,8 & 38,0 & 6,0 & 6,0 & 3 & 42,5 & 42,5 & 5,4 & 5,5 \\
\hline 2 & 43,1 & 40,0 & 6,1 & 6,0 & 3 & 41,8 & 42,3 & 5,2 & 5,3 \\
\hline
\end{tabular}


ANEXO: Dados de resistência à compressão $\left(f_{c}\right)$ e à tração na flexão $\left(f_{t}\right)$ dos três conjuntos amostrais utilizados no estudo (cont.).

\begin{tabular}{ccccc}
\hline Amostra & $\mathbf{f}_{\mathrm{c} 1}$ & $\mathbf{f}_{\mathrm{c} 2}$ & $\mathbf{f}_{\mathbf{t} 1}$ & $\mathbf{f}_{\mathbf{t} 2}$ \\
\hline 2 & 40,4 & 40,0 & 6,3 & 6,0 \\
\hline 2 & 40,1 & 37,0 & 6,0 & 5,8 \\
\hline 2 & 37,6 & 38,0 & 5,6 & 5,7 \\
\hline 2 & 36,6 & 37,9 & 5,8 & 5,8 \\
\hline 2 & 38,0 & 37,1 & 5,6 & 5,0 \\
\hline 2 & 39,0 & 38,1 & 5,6 & 5,7 \\
\hline 2 & 38,1 & 37,0 & 5,3 & 6,0 \\
\hline 2 & 39,2 & 38,0 & 5,2 & 5,5 \\
\hline 2 & 36,8 & 37,0 & 5,5 & 5,6 \\
\hline 2 & 38,1 & 38,0 & 5,2 & 6,1 \\
\hline 2 & 36,0 & 36,1 & 5,6 & 5,6 \\
\hline 2 & 37,3 & 36,8 & 5,5 & 6,6 \\
\hline 2 & 38,2 & 38,1 & 5,2 & 5,2 \\
\hline 2 & 35,6 & 36,0 & 5,9 & 5,8 \\
\hline
\end{tabular}

\begin{tabular}{ccccc}
\hline Amostra & $\mathbf{f}_{\mathrm{c} 1}$ & $\mathbf{f}_{\mathrm{c} 2}$ & $\mathbf{f}_{\mathbf{t} 1}$ & $\mathbf{f}_{\mathbf{t} 2}$ \\
\hline 3 & 40,6 & 38,7 & 6,3 & 6,6 \\
\hline 3 & 39,0 & 41,3 & 5,5 & 5,7 \\
\hline 3 & 44,6 & 45,3 & 6,6 & 6,0 \\
\hline 3 & 38,8 & 40,8 & 5,3 & 6,0 \\
\hline 3 & 40,0 & 43,6 & 5,3 & 5,7 \\
\hline 3 & 41,3 & 42,7 & 6,0 & 5,2 \\
\hline 3 & 38,3 & 39,5 & 5,7 & 5,7 \\
\hline 3 & 39,0 & 45,0 & 5,8 & 6,4 \\
\hline 3 & 39,6 & 42,8 & 5,9 & 5,6 \\
\hline 3 & 44,6 & 40,0 & 5,4 & 5,4 \\
\hline 3 & 41,4 & 43,8 & 5,7 & 5,4 \\
\hline 3 & 43,6 & 43,0 & 5,7 & 5,4 \\
\hline 3 & 42,9 & 42,8 & 5,0 & 5,7 \\
\hline 3 & 43,2 & 43,0 & 5,9 & 5,4 \\
\hline & & & &
\end{tabular}

